Pacific Journal of Mathematics

ON COMPLETENES 


\title{
ON COMPLETENESS
}

\author{
NORMaN R. Howes
}

Hisahiro Tamano asked the following question that motivated his Some properties of the Stone-Cech compactification, J. Math. Soc. Japan, vol. 12, 1 (1960) 104-117. "What is a necessary and sufficient condition for a uniform space to have a paracompact completion?"'. Although he did not arrive at a solution, he obtained characterizations of completeness, paracompactness, and the structure of the completion of a uniform space by means of the radical.

This paper provides an answer to the above question and related ones. Also included is a development of other types of completeness in both uniform and non-uniform topological spaces. These other "complete" spaces yield characterizations of paracompactness and the Lindelöf property as well as necessary and sufficient conditions for paracompact spaces to be Lindelöf and entirely normal spaces to be paracompact.

Cofinal completeness.

In what follows $(X, \mu)$ denotes a uniform space in the sense of Tukey [13]; ie, $\mu$ will be a filter of coverings with respect to $<_{*}$ (star refinement). A covering $\mathscr{V}^{-}$is said to star refine a covering $\mathscr{U}$ if for each $V \in \mathscr{V}^{-}$there exists a $U \in \mathscr{C}$ such that $\operatorname{Star}(V, \mathscr{V}) \subset$ $U$ where $\operatorname{Star}\left(V, \mathscr{V}^{-}\right)=U\left\{V \in \mathscr{V}^{-} \mid W \cap V \neq \phi\right\}$. This development of uniform spaces is equivalent to the one given by Bourbaki [1]; confer Isbell [6] p. 12.

A net $\psi: D \rightarrow X$ is said to be cofinally Cauchy if for each $\mathscr{U} \in \mu$ there is a cofinal $C \subset D$ such that $\psi(C) \subset U$ for some $U \in \mathscr{Q}$. We will call a uniform space $(X, \mu)$ cofinally complete if each cofinally Cauchy net quasi-converges (is frequently in each neighborhood of some point $p$ ). When a net quasi-converges to some point $p, p$ is often called a cluster point of the net.

A family of coverings $\nu$ in which every member of $\nu$ has a star refinement in $\nu$ is said to be a normal family. Since every collection $\lambda$ of coverings contains a largest normal family $\mu$ we say the members of $\mu$ are normal in $\lambda$. A covering $\mathscr{C}$ is said to be normal with respect to $\lambda$ if it is normal in $\kappa$ where $\kappa$ is the collection of all open coverings that are refined by members of $\lambda$.

Given a completely regular topology $\tau$ there exists a finest uniformity $u$ for $\tau$. It consists of all coverings that are normal with respect to the family of all open coverings. The open members of $u$ are called normal coverings, and $u$ is called the universal uniformity. A space equipped with the universal uniformity is often 
called a fine uniform space.

If $X$ is a completely regular $T_{1}$ space there is a uniformity $\beta$ for $X$ that has a basis which consists of all finite normal coverings. The completion of $(X, \beta)$ is $\beta X$, the Stone-Cech compactification of $X$. Shirota [10] showed that every completely regular $T_{1}$ space has a uniformity that has a basis consisting of all countable normal coverings. He called this uniformity the e-uniformity and proved the following:

THEOREM. For a completely regular space $X$ the following are equivalent:

(1) $X$ is e-complete (complete with respect to the $e$-uniformity),

(2) $X$ is realcompact ( $X$ is a $Q$-space see Hewitt [5]), and

(3) $X$ is homeomorphic to a closed subspace of a product of real lines.

A directed set $D$ is said to be $\omega$ directed if for each $\left\{d_{i}\right\}_{i=1}^{\infty}$ which is a subset of $D$ there is a $d \in D$ such that $d_{i} \leqq d$ for each $i$. A net $\psi: D \rightarrow X$ is $\omega$ directed if $D$ is an $\omega$ directed set. The following theorem shows that cofinal completeness of the $e$-uniformity is also of some interest.

THEOREM 1. In a completely regular space $X$ the following are equivalent:

(1) $X$ is Lindelöf,

(2) $X$ is cofinally e-complete, and

(3) each $\omega$ directed net in $X$ quasi-converges.

Proof. (1) $\rightarrow$ (2) Let $\psi: D \rightarrow X$ be a cofinally Cauchy net with respect to the $e$-uniformity and suppose $\psi$ does not quasi-converge. Then for each $p \in X$ there is an open set $V(p)$ containing $p$ such that $\psi$ is eventually in $X-V(p)$. Put $\mathscr{Y}^{-}=\{V(p) \mid p \in X\}$. Then $\mathscr{y}$ has a countable subcovering $\left\{V\left(p_{i}\right)\right\}_{i=1}^{\infty}$. Since $X$ is regular and Lindelö it is also paracompact so that the finest uniformity for $X$ consists of all open coverings. But then $\left\{V\left(p_{i}\right)\right\}_{i=1}^{\infty}$ is a normal covering and hence a member of $e$. But then $\psi$ cannot be frequently in some member of $\left\{V\left(p_{i}\right)\right\}_{1=1}^{\infty}$ since it is eventually in $X-V\left(p_{i}\right)$ for each $i$. Therefore $\psi$ is not cofinally Cauchy with respect to $e$ which is a contradiction. Consequently $\psi$ must quasi-converge.

$(2) \rightarrow(3)$ Let $\psi: D \rightarrow X$ be an $\omega$ directed net and let $\mathscr{U} \in e$. Then there is a countable covering $\mathscr{Y}=\left\{V_{i}\right\}_{i=1}^{\infty}$ such that $\mathscr{y}^{\infty} \in e$ and $\mathscr{V}$ refines $\mathscr{U}\left(\mathscr{V}^{-}<\mathscr{C}\right)$. Suppose $\psi$ is not frequently in some member of $\mathscr{Y}$ and put $\left.D_{i}=d \in D \mid \psi(d) \in V_{i}\right\}$. Then there exists a $d_{i} \in D$ such that $d \leqq d_{i}$ for each $d \in D_{i}$ or else $D_{i}$ would be frequently 
in $V_{i}$. Since $\psi$ is $\omega$ directed there is a $d \in D$ such that $d_{i} \leqq d$ for each $i$. Now $\psi(d) \in V_{j}$ for some $j$ since $\mathscr{Y}$ covers $X$. Hence $d \in D_{j}$ which implies $d<d_{j}$. But $d_{j} \leqq d$ which is a contradiction. Therefore $\psi$ must frequently be in some member of $\mathscr{V}$. Since $\mathscr{V}$ was chosen arbitrarily, $\psi$ is cofinally Cauchy with respect to $e$ and therefore quasi-converges.

$(3) \rightarrow(1)$ If $X$ is not Lindelöf there is a covering $\mathscr{Q}$ of $X$ having no countable subcovering. For each countable $B \subset \mathscr{Q}$ put $V(B)=$ $\mathrm{U}\{U \mid U \in B\}$ and $F(B)=X-V(B)$. Let $S$ be the set of all countable subsets of $\mathscr{U}$ and for each $B \in S$ pick $\psi(B) \in F(B)$. The assignment $B \rightarrow \psi(B)$ defines an $\omega$-directed net $\psi: S \rightarrow X$ that quasi-converges to some $p \in X$ where $S$ is directed by set inclusion. Let $U \in \mathscr{U}$ such that $p \in U$. Then $\psi$ is eventually in $F(\{U\})$ and hence cannot be frequently in $U$ which is a contradiction. Consequently $X$ is Lindelöf.

H. H. Corson [3] showed that a completely regular space is Lindelöf if and only if each filter which is weakly Cauchy with respect to the coursest uniformity $\lambda$, such that all real valued continuous functions are uniformly continuous, has a cluster point. Hence Theorem 1. suggests a connection between cofinal completeness and the property that each weakly Cauchy filter clusters. This connection is clarified in the following:

Lemma. Each weakly Cauchy filter in a uniform space $(X, \mu)$ clusters if and only if $(X, \mu)$ is cofinally complete.

Proof. Corson defines a filter $\mathscr{F}$ to be weakly Cauchy if for each $\mathscr{U} \in \mu$ there is a filter $\mathscr{G}$ containing $\mathscr{F}$ and a $G \in \mathscr{G}$ such that $G \subset U$ for some $U \in \mathscr{Q}$.

(Necessity) Let $\psi: D \rightarrow X$ be a cofinally Cauchy net. Put $\mathscr{G}=$ $\{G \subset X \mid \psi$ is eventually in $G\}$. Pick $\mathscr{Q} \in \mu$ and a cofinal $C \subset D$ with $\psi(C) \subset U$ for some $U \in \mathscr{Q}$. Let $\mathscr{F}=\left\{F \subset X \mid \psi_{0}\right.$ is eventually in $\left.F\right\}$ where $\psi_{c}$ denotes $\psi$ restricted to $C$. Since $\mathscr{G} \subset \mathscr{F}$ and $\mathscr{F}$ contains a set $H$ such that $H \subset U, \mathscr{G}$ is weakly Cauchy and therefore has a cluster point $p$. Then $p \in \overline{\psi(R)}$ for each residual $R \subset D$ which implies $\psi$ quasi-converges to $p$.

(Sufficiency) Suppose $(X, \mu)$ is cofinally complete and $\mathscr{F}$ is a weakly Cauchy filter. Let $S=\{(x, F) \mid F \in \mathscr{F}$ and $x \in F\}$. Put $(x, F) \leqq$ $(\mathrm{y}, G)$ if $G \subset F$ and let $\psi: S \rightarrow X$ be the net defined by $\psi(x, F)=x$. If $\mathscr{U} \in \mu$ there is filter $\mathscr{H}$ containing $\mathscr{F}$ such that $H \subset U$ for some $H \in \mathscr{H}$ and $U \in \mathscr{U}$. Let $F \in \mathscr{F}$ and put $C=\{(x, G) \in S \mid x \in H \cap F\}$. Then $\psi(C) \subset U$ and $C$ is cofinal in $S$. Hence $\psi$ is cofinally Cauchy and therefore quasi-converges to some $p \in X$. But then $p$ is a cluster point of $\mathscr{F}$. 
Corson also showed in [3] that a $T_{1}$ space is paracompact if and only if there exists a uniformity for $X$ such that all weakly Cauchy filters cluster. Thus the preceding lemma together with Corson's theorem shows that a $T_{1}$ space $X$ is paracompact if and only if there is a uniformity $\mu$ for $X$ such that $(X, \mu)$ is cofinally complete. This leads to the following observation:

TheoReM 2. For a completely regular $T_{1}$ space $X$ we have:

(1) $X$ is paracompact if and only if $X$ is cofinally complete with respect to the $u$-uniformity.

(2) $X$ is Lindelöf if and only if $X$ is cofinally complete with respect to the e-uniformity, and

(3) $X$ is compact if and only if $X$ is cofinally complete with respect to the $\beta$-uniformity.

Proof. The proof of (1) is contained in the preceding remarks and the proof of (2) is part of Theorem 1. To prove (3) suppose $X$ is compact which implies each net has a convergent subnet which is equivalent to each net quasi-converging. Hence $X$ is cofinally complete with respect to the $\beta$ uniformity.

Conversely assume $X$ is cofinally complete with respect to the $\beta$ uniformity. Let $\psi: D \rightarrow X$ be a net and let $\mathscr{V} \in \beta$. Then there exists a refinement $\mathscr{U}$ of $\mathscr{C}$ such that $\mathscr{C}=\left\{U_{i}\right\}_{i=1}^{n}$ for some positive integer $n$. Put $D_{i}=\left\{d \in D \mid \psi(d) \in U_{i}\right\}$. Assume no $D_{i}$ is cofinal in $D$ which implies that for each $i=1, \cdots, n$ there is a $d_{i} \in D$ such that $d \leqq$ $d_{i}$ for each $d \in D_{i}$. Pick $d_{0} \in D$ such that $d_{i} \leqq d_{0}$ for each $i=1, \cdots, n$. Then $d \leqq d_{0}$ for each $d \in D$ which implies $d_{0}$ is a last point of $D$. But $d_{0} \in D$ implies $\psi\left(d_{0}\right) \in X$ which in ture implies $\psi\left(d_{0}\right) \in U_{j}$ for some positive integer $j$ such that $1 \leqq j \leqq n$. But then $d_{0} \in D_{j}$ which implies $D_{j}$ is cofinal in $D$ after all. Hence $\psi$ is cofinally Cauchy which implies $\psi$ quasi-converges. But then $\psi$ has a convergent subset which implies $X$ is compact.

We are now in a position to give a necessary and sufficient condition for a uniform space to have a paracompact completion.

We define a uniform space to be preparacompact if each cofinally Cauchy net has a Cauchy subnet. In the following theorem we will also be determining a necessary and sufficient condition for a uniform space to have a Lindelöf completion. Hence, the following definitions: We will call a uniform space countably bounded if each uniform covering has a countable subcovering and pre-Lindelöf if it is both countably bounded and preparacompact.

Finally we define the derived uniformity. Let $(X, \mu)$ be a uniform space and $(\tilde{X}, \tilde{\mu})$ be its completion. Let $\tilde{u}$ be the universal 
uniformity for $\tilde{X}$ and let $\nu$ be the uniformity induced on $X$ by $\tilde{u}$. Then $\nu$ is called the uniformity derived from $\tilde{u}$ or simply the "derived" uniformity.

THEOzEM 3. Let $(X, \mu)$ be a uniform space and $\nu$ be the derived uniformity. Then:

(1) $(X, \mu)$ has a paracompact completion if and only if $(X, \mu)$ is preparacompact,

(2) $(X, \mu)$ has a Lindelöf completion if and only if $(X, \nu)$ is pre-Lindelöf, and

(3) $(X, \mu)$ has a compact completion if and only if $(X, \nu)$ is precompact.

Proof of (1). Let $(\tilde{X}, \tilde{\mu})$ be the completion of $(X, \mu)$ and let $\tilde{u}$ be the universal uniformity for $\tilde{X}$. Then $(X, \nu)$ is a dense uniform subspace of $(\widetilde{X}, \widetilde{u})$. Assume $(X, \nu)$ is preparacompact and that $\psi: D \rightarrow$ $\widetilde{X}$ is cofinally Cauchy with respect to $\tilde{u}$. Since $(\tilde{X}, \tilde{\mu})$ is complete, so is $(\tilde{X}, \widetilde{u})$. Let $E=D \times \tilde{u}$ and define $\leqq$ on $E$ by $(d, \widetilde{\mathscr{U}}) \leqq(e, \widetilde{\mathscr{V}})$ if $d \leqq e$ and $\widetilde{\mathscr{V}}<* \widetilde{\mathscr{C}}$. For each $(d, \widetilde{\mathscr{C}}) \in E$ put $\theta(d, \widetilde{\mathscr{C}})=a$ for some $a \in X$ such that $a$ and $\psi(d)$ both belong to some $\widetilde{U} \in \widetilde{\mathscr{C}}$. Then the correspondence $(d, \widetilde{\mathscr{C}}) \rightarrow \theta(d, \widetilde{\mathscr{C}})$ defines a net $\theta: E \rightarrow X$.

Let $\tilde{\mathscr{U}} \in \tilde{u}$ and pick $\tilde{\mathscr{V}} \in \tilde{u}$ with $\tilde{\mathscr{V}}<* \widetilde{\mathscr{U}}$. Since $\psi$ is cofinally Cauchy there is a cofinal $C \subset D$ with $\psi(C) \subset \tilde{V}$ for some $\tilde{V} \in \widetilde{\mathscr{V}}$. Put

$$
A=\{(d, \widetilde{\mathscr{W}}) \mid d \in C \text { and } \widetilde{\mathscr{W}}<* \widetilde{\mathscr{V}}\} .
$$

Then $A$ is cofinal in $E$. Let $(d, \widetilde{\mathscr{W}}) \in A$. Then $\theta(d, \widetilde{\mathscr{W}})=y \in X$ such that $y$ and $\psi(d)$ both belong to some $\widetilde{W} \in \widetilde{\mathscr{W}}$. Since $(d, \widetilde{\mathscr{W}}) \in A$, $d \in C$ which puts $\psi(d)$ in $\tilde{V}$. Consequently we have:

$$
y \in \operatorname{Star}(\widetilde{V}, \widetilde{\mathscr{W}}) \subset \operatorname{Star}(\widetilde{V}, \widetilde{\mathscr{V}}) \subset \widetilde{U}
$$

for some $\tilde{U} \in \widetilde{\mathscr{C}}$. Therefore $\theta(A) \subset \widetilde{U}$ which implies $\theta$ is cofinally Cauchy in $(\tilde{X}, \widetilde{u})$. But $\theta(E) \subset X$ implies $\theta$ is cofinally Cauchy in $(X, \nu)$. Consequently $\theta$ has a Cauchy subnet $\xi: K \rightarrow X$. But then $\xi$ is Cauchy in $\widetilde{X}$ and hence converges to some $x \in \tilde{X}$. Therefore $\theta$ quasi-converges to $x$. It remains to show that $\psi$ quasi-converges to $x$. For this let 0 be an open set containing $x$. Then there is a $\widetilde{\mathscr{U}} \in \tilde{u}$ such that $x \in \operatorname{Star}(x, \widetilde{\mathscr{C}}) \subset 0$ where the members of $\widetilde{\mathscr{C}}$ are open sets. Pick $\widetilde{\mathscr{V}} \in \tilde{u}$ such that $\widetilde{\mathscr{V}}<* \widetilde{\mathscr{U}}$. Let $S$ be cofinal in $E$ such that $\theta(S) \subset \widetilde{V}$ for some $\tilde{V} \in \widetilde{\mathscr{V}}$ containing $x$. Put 


$$
D(S)=\{d \in D \mid(d, \widetilde{\mathscr{W}}) \in S \text { for some } \widetilde{\mathscr{W}}<* \widetilde{\mathscr{V}}\}
$$

Then $D(S)$ is cofinal in $D$. For each $d \in D(S), \psi(d)$ and $\theta(d, \widetilde{\mathscr{W}})$ are contained in some $\widetilde{W} \in \widetilde{\mathscr{W}}$ for some $(d, \widetilde{\mathscr{V}}) \in S$ where $\widetilde{\mathscr{V}}<* \widetilde{\mathscr{V}}$, which implies $\psi(d)$ and $\theta(d, \widetilde{\mathscr{V}})$ are contained in some $V_{1} \in \widetilde{\mathscr{V}}$ for each $(d, \widetilde{\mathscr{W}}) \in S$. But $(d, \widetilde{\mathscr{W}}) \in S$ implies $\theta(d, \widetilde{\mathscr{W}}) \in \widetilde{V}$. Hence

$$
\psi(d) \in \tilde{V} \cup \widetilde{V}_{1} \subset \operatorname{Star}(\tilde{V}, \widetilde{\mathscr{V}}) \subset \tilde{U}
$$

for some $\tilde{U} \in \tilde{\mathscr{C}}$. But $x \in \tilde{V}$ implies $x \in \tilde{U}$. Then $\tilde{U} \subset 0$ since $\operatorname{Star}(x, \widetilde{\mathscr{C}}) \subset 0$. Consequently $\psi(d) \in 0$ for each $d \in D(S)$ which implies $\psi$ quasi-converges to $x$. Therefore each cofinally Cauchy net in $\widetilde{X}$ quasi-converges which implies $(\widetilde{X}, \widetilde{u})$ is cofinally complete. But then $\widetilde{X}$ is paracompact by Theorem 2 .

Conversely suppose $\tilde{X}$ is paracompact. By Theorem $2(\widetilde{X}, \widetilde{u})$ is cofinally complete. Let $\psi: D \rightarrow X$ be cofinally Cauchy with respect to $\nu$. Since $(X, \nu)$ is a uniform subspace of $(\widetilde{X}, \widetilde{u})$, we know that $\psi$ is cofinally Cauchy in $(\tilde{X}, \tilde{u})$. Also since $(\tilde{X}, \widetilde{u})$ is cofinally complete, $\psi$ quasi-converges to some $p \in \tilde{X}$. But then $\psi$ has a subnet $\theta$ that converges to $p$. Then $\theta$ is Cauchy in $(\tilde{X}, \widetilde{u})$. But $\theta \subset X$ and therefore $\theta$ is Cauchy in $(X, \nu)$. Consequently, each cofinally Cauchy net in $(X, \nu)$ has a Cauchy subnet so that $(X, \nu)$ is preparacompact.

Proof of (2). Assume first that $\tilde{X}$ is Lindelöf. Then $\tilde{X}$ is paracompact and hence $(\tilde{X}, \widetilde{u})$ is cofinally complete. But then $(X, \nu)$ is preparacompact as was shown in part (1). Next let $\mathscr{Y} \in \nu$. Then $\mathscr{V}$ has a uniform refinement $\mathscr{U}$ consisting of closed sets. For each $U \in \mathscr{C}$ put $\widetilde{U}=\mathrm{Cl}_{\tilde{X}}(U)$ and let $\widetilde{\mathscr{U}}=\{\widetilde{U} \mid \widetilde{U} \in \mathscr{C}\}$. Then $\mathscr{C} \in \widetilde{u}$ and hence has a countable subcovering say $\left\{\widetilde{U}_{i}\right\}$. Then $\left\{U_{i}\right\}$ covers $X$. In fact, if $p \in X$ then $p \in \widetilde{X}$ which implies $p \in \widetilde{U}_{j}$ for some positive integer $j$. Hence $p \in \mathrm{Cl}_{\widetilde{X}}\left(U_{j}\right)$. Let 0 be open in $X$ such that $p \in 0$. Then $0=\tilde{0} \cap X$ for some $\tilde{0}$ that is open in $\tilde{X}$. Now $p \in \tilde{0}$ which implies $\tilde{0} \cap U_{j} \neq \phi$ since $p \in \mathrm{Cl}_{\widetilde{X}}\left(U_{j}\right)$. Then there is a $t \in \tilde{0} \cap \widetilde{U}_{j}$. $t \in U_{j}$ implies $t \in X$ so we have $(\tilde{0} \cap X) \cap U_{j} \neq \phi$. Hence $0 \cap U_{j} \neq \phi$ so that $p \in \mathrm{Cl}_{X}\left(U_{j}\right)=U_{j}$. Since $\left\{U_{i}\right\}$ covers $X$ there exists some $\left\{V_{i}\right\} \subset \mathscr{V}$ such that $\bigcup V_{i}=X$. Consequently $X$ is countably bounded and hence pre-Lindelöf.

Conversely assume $(X, \nu)$ is pre-Lindelöf which implies $(X, \nu)$ is preparacompact and countable bounded. Since $(X, \nu)$ is preparacompact $(\tilde{X}, \tilde{\mu})$ is paracompact by part $(1)$ so that $(\tilde{X}, \widetilde{u})$ is cofinally complete by Theorem 2. Let $\widetilde{\mathscr{Q}} \in \widetilde{u}$. Since $\tilde{X}$ is paracompact there exists a locally finite open refinement $\widetilde{\mathscr{V}}^{-}=\left\{\tilde{V}_{\beta} \mid \beta \in B\right\}$. Since $X$ is normal we can shrink $\widetilde{\mathscr{V}}$ to an open covering $\widetilde{\mathscr{W}}=\left\{\widetilde{W}_{\beta} \mid \beta \in B\right\}$ such that 
$\mathrm{Cl}_{\widetilde{X}}\left(\widetilde{W}_{\hat{f}}\right) \subset \widetilde{V}_{\beta}$ for each $\beta \in B$. Then $\widetilde{\mathscr{W}}$ is also locally finite. Since $\widetilde{\mathscr{W}}$ is an open covering in a paracompact space $\widetilde{X}$, it must be a member of the universal uniformity $\tilde{u}$. But then $\mathscr{W}=\{\widetilde{W} \cap X \mid \widetilde{W} \in \widetilde{\mathscr{W}}\}$ belongs to $\nu$ which implies there is a countable subcovering $\left\{W_{\beta_{i}}\right\} \subset \mathscr{W}$. Since $\widetilde{\mathscr{V}}$ is locally finite in $\widetilde{X},\left\{W_{\beta_{i}}\right\}$ is locally finite in $\widetilde{X}$. Hence $\mathrm{U}_{i=1}^{\infty} \mathrm{Cl}_{\widetilde{X}}\left(W_{\beta_{i}}\right)=\mathrm{Cl}_{\widetilde{X}}\left(\bigcup_{i=1}^{\infty} W_{\beta_{i}}\right)=\mathrm{Cl}_{\widetilde{X}}(X)=\widetilde{X}$ since $X$ is dense in $\tilde{X}$. Thererfore $\left\{\mathrm{Cl}_{\widetilde{X}}\left(W_{\beta_{i}}\right)\right\}$ covers $X$ and

$$
\mathrm{Cl}_{\widetilde{X}}\left(W_{\beta_{i}}\right) \subset \mathrm{Cl}_{\widetilde{X}}\left(\widetilde{W}_{\beta_{i}}\right) \subset \widetilde{V}_{\beta_{i}}
$$

for each positive integer $i$. Hence $\left\{\widetilde{V}_{\beta_{i}}\right\}$ covers $\widetilde{X}$. But since $\widetilde{\mathscr{Y}}$ refines $\widetilde{\mathscr{C}}$ there exists a countable subcovering $\left\{\widetilde{U}_{\beta_{i}}\right\} \subset \widetilde{\mathscr{C}}$ that covers $\tilde{X}$. Therefore $(\widetilde{X}, \widetilde{u})$ is also countably bounded. We are now in a position to show that $\tilde{X}$ is Lindelö. We will use the fact that $(\widetilde{X}, \widetilde{u})$ is cofinally complete and countably bounded to show that each $\omega$-directed net in $\tilde{X}$ quasi-converges. We will then invoke Theorem 1 to obtain the desired result. Let $\psi: D \rightarrow \widetilde{X}$ be an $\omega$-directed net and let $\widetilde{\mathscr{C}} \in \tilde{U}$. Then $\widetilde{\mathscr{C}}_{\mathscr{C}}$ has a countable subcovering say $\left\{\widetilde{U}_{i}\right\}$. Put $D_{i}=\left\{d \in D \mid \psi(d) \in \widetilde{U}_{i}\right\}$ for each $i$ and suppose $D_{i}$ is not cofinal in $D$ for each $i$. Then there exists a $d_{i} \in D$ for each $i$ such that $d \leqq d_{i}$ for each $d \in D_{i}$. Since $\psi$ is $\omega$-directed there is a $d_{0} \in D$ such that $d_{i} \leqq d_{0}$ for each $i$. Since $\left\{\widetilde{U}_{i}\right\}$ covers $\widetilde{X}, \psi\left(d_{0}\right) \in \widetilde{U}_{j}$ for some positive integer $j$ which implies $d_{0} \in D_{j}$ which in turn implies $D_{j}$ is cofinal in $D$ since $D=\bigcup_{i=1}^{\infty} D_{i}$. Therefore $\psi$ is frequently in $U_{j}$. Hence $\psi$ is cofinally Cauchy and consequently must quasi-converge. Thus $\widetilde{X}$ is Lindelöf by Theorem 1 .

Proof of (3). It is well known that $(X, \mu)$ has a compact completion $(\tilde{X}, \tilde{\mu})$ if and only if $\mu$ is precompact. But if $\nu$ is precompact then $\mu \subset \nu$ implies $\mu$ is precompact which in turn implies $\widetilde{X}$ is compact.

On the other hand, if $\widetilde{X}$ is compact then $\tilde{\mu}=\widetilde{u}$ which implies $\mu=\nu$ and $\mu$ is precompact which implies $\nu$ is precompact.

Theorem 3 has several corollaries. The first is merely a special case of Theorem 3 when $X$ is equipped with the $u$-uniformity; i.e., when $X$ is a fine uniform space.

COROLlary 1. Let $u$ be the universal uniformity for a completely regular $T_{1}$ space $X$. Then:

(1) $(X, u)$ has a paracompact completion if and only if it is preparacompact,

(2) $(X, u)$ has a Lindelöf completion if and only if it is preLindelöf, and 
(3) $(X, u)$ has a compact completion if and only if it is precompact.

As is well known, (3) of the above corollary is not dependent upon $X$ having the $u$-uniformity. The following corollary could actually be considered a lemma in the proof of Theorem 3, and is recorded here because it is of sufficient interest itself. It provides us an analogy with precompactness; i.e., both precompactness and preparacompactness are properties that are carried over into the completion.

COROLLARY 2. The completion of a preparacompact uniform space is cofinally complete (and hence paracompact and pre-paracompact).

COROLlary 3. A countably bounded cofinally complete uniform space is Lindelöf.

CoRollary 4. A paracompact space is Lindelöf if and only if it is countably bounded with respect to the universal uniformity.

Additional results along these lines can be obtained and may be of some interest. We state two of them below without proof.

Proposition 1. A completely regular $T_{1}$ space is paracompact if and only if it is complete and preparacompact with respect to the universal uniformity.

Proposition 2. A uniform space $(X, \mu)$ is countably bounded if and only if each $\omega$-directed net is cofinally Cauchy.

Cofinal $\Delta$ completeness. A net $\psi: D \rightarrow X$ in a topological space $X$ will be called cofinally $\triangle$ Cauchy if for each open covering $\mathscr{U}$ of $X$ there is a $p \in X$ and a cofinal $C \subset D$ such that $\psi(C) \subset \operatorname{Star}(p, \mathscr{U})$. $X$ will be called cofinally $\Delta$ complete if each cofinally $\triangle$ Cauchy net quasi-converges.

A space is said to be entirely normal if the collection of all neighborhoods of the diagonal in $X \times X$ forms a uniformity for $X$ in the sense of Bourbaki [1]. Mansfield [8] calls a space $X$ almost2-fully normal if for each open covering $\mathscr{C}$ of $X$ there is an open refinement $\mathscr{V}$ of $\mathscr{Q}$ such that if $p \in V \in \mathscr{Y}, q \in W \in \mathscr{Y}$, and $V \cap W \neq$ $\phi$ then there exists a $U \in \mathscr{C}$ containing both $p$ and $q . \mathrm{H}$. J. Cohen [2] showed that entire normality and almost-2-full normality are equivalent properties. 
Proposition 3. An entirely normal space is paracompact if and only if it is cofinally $\Delta$ complete.

Proof. A simple proof of the necessity can be obtained after proving Proposition 4, since a paracompact space is known to be both entirely normal and metacompact.

The sufficiency part of the proof will consist of showing that in an entirely normal space, the cofinally Cauchy nets are also cofinally $\Delta$ Cauchy and hence when entire normality is encountered in the presence of cofinal $\Delta$ completeness we get cofinal completeness and hence paracompactness.

Let $X$ be entirely normal and let $\mathscr{C}$ be the uniformity consisting of all neighborhoods of the diagonal. For each $V \in \mathscr{M}$ put $\mathscr{V}=\{V[x] \mid x \in X\}$. Then $\mu=\{\mathscr{V} \mid V \in \mathscr{l}\}$ is a covering uniformity for $X$. Let $\mathscr{C}$ be an open covering of $X$ and let $W=U\{U \times U \mid U \in \mathscr{C}\}$. Then $W \in \mathscr{M}$. Hence there exists a $V \in \mathscr{C}$ with $V \circ V \subset W$ and $V=$ $V^{-1}$. Let $p \in X$. Then $V[p]=\{x \in X \mid(p, x) \in V\}=\{x \in X \mid(x, p) \in V\}=$ $\{x \in X \mid p \in V[x]\} \subset \operatorname{Star}(p, \mathscr{V})$. Let $y \in \operatorname{Star}(p, \mathscr{Y})$ which implies there is a $q \in X$ such that $y \in V[q]$ and $p \in V[q]$ which in turn implies that $(q, p),(q, y) \in V$. But then $(p, q),(q, y) \in V$ which implies $(p, y) \in V \circ V \subset W$. Hence $(p, y) \in U \times U$ for some $U \in \mathscr{Q}$ which implies $p, y \in U$ and hence $y \in \operatorname{Star}(p, \mathscr{C})$. Therefore $V[p] \subset \operatorname{Star}(p, \mathscr{V}) \subset \operatorname{Star}(p, \mathscr{C})$.

Let $\psi: D \rightarrow X$ be cofinally Cauchy with respect to $\mu$. Then there exists a cofinal $C \subset D$ such that $\psi(C) \subset V[z]$ for some $z \in X$. But then $\psi(C) \subset \operatorname{Star}(p, \mathscr{U})$. Therefore $\psi$ quasi-converges. Hence $(X, \mu)$ is paracompact.

Finally we observe the following:

Proposition 4. A metacompact space is cofinally 4 complete.

This gives us the following diagram:

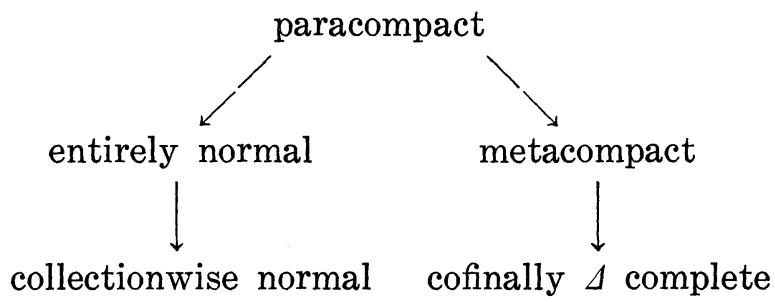

E. Michael [9] showed that collectionwise normality and metacompactness is a factorization of paracompactness; i.e., both are conditions strictly weaker than paracompactness such that the pair of conditions together is a sufficient condition for paracompactness. Our Proposition 4 shows that entire normality and cofinal $\Delta$ com- 
pleteness is a similar factorization.

Added Note. Kitti Morita, in his paper entitled Topological completions and $M$-spaces presented at the 1970 Pittsburgh Topology conference, listed five unsolved problems in Section 7. It should be noted that our Corollary 1 provides an answer to the first and third parts of Morita's First Problem.

\section{REFERENCES}

1. N. Bourbaki, Topologie générale, Paris, 1951.

2. H. J. Cohen, Sur un problème de M. Dieudonné, C. R. Acad. Sci. Paris, 234 (1952), 290-292.

3. H. H. Corson, The determination of paracompactness by uniformities, Amer.

J. Math., 80 (1959), 185-190.

4. ___ Normality in subsets of product spaces, Amer. J. Math., 81 (1959), 785-796.

5. E. Hewitt, Rings of real-valued continuous functions I, Trans. Amer. Math. Soc., 64 (1948), 45-99.

6. J. R. Isabell, Uniform Spaces, Providence, 1964.

7. J. Kelley, General Topology, New York, 1955.

8. M. J. Mansfield, Some generalizations of full normality, Trans. Amer. Math. So., 86 (1957), 489-505.

9. E. Michael, Point finite and locally finite coverings, Can. J. Math., 7 (1955), 275-279.

10. T. Shirota, A class of topological spaces, Osaka. Math. J., 4 (1952), 23-40.

11. A. H. Stone, Paracompactness and product spaces, Bull. Amer. Math. Soc., 54 (1948), 977-982.

12. H. Tamano, Some properties of the Stone-Čech compactification, J. Math. Soc. Japan, 12, 1 (1960), 104-117.

13. J. W. Tukey, Convergence and uniformities in topology, Princeton, 1940.

14. A. Weil, Sur les espaces à structure uniforme et sur la topologie générale, Paris, 1937.

Received March 2, 1970 and revised form September 27, 1970. The author is deeply indebted to the late Prof. Tamano not only for communicating this problem during a seminar at Texas Christian University in 1968-69 but also for his kind guidance and encouragement while working on a dissertation under him during 1967-68.

UNIVERSITY OF DALLAS

AND

Texas Instruments, Inc. 


\section{PACIFIC JOURNAL OF MATHEMATICS}

\section{EDITORS}

\author{
H. SAMELSON \\ Stanford University \\ Stanford, California 94305 \\ C. R. HовBY \\ University of Washington \\ Seattle, Washington 98105
}

J. DUGUndis

Department of Mathematics

University of Southern California

Los Angeles, California 90007

RICHARD ARENS

University of California

Los Angeles, California 90024

\section{ASSOCIATE EDITORS}
E. F. BeCKenbaCH
B. H. NeUmanN
F. WOLF
K. YoshidA

\section{SUPPORTING INSTITUTIONS}

\author{
UNIVERSITY OF BRITISH COLUMBIA \\ CALIFORNIA INSTITUTE OF TECHNOLOGY \\ UNIVERSITY OF CALIFORNIA \\ MONTANA STATE UNIVERSITY \\ UNIVERSITY OF NEVADA \\ NEW MEXICO STATE UNIVERSITY \\ OREGON STATE UNIVERSITY \\ UNIVERSITY OF OREGON \\ OSAKA UNIVERSITY \\ UNIVERSITY OF SOUTHERN CALIFORNIA
}

\author{
STANFORD UNIVERSITY \\ UNIVERSITY OF TOKYO \\ UNIVERSITY OF UTAH \\ WASHINGTON STATE UNIVERSITY \\ UNIVERSITY OF WASHINGTON

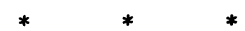 \\ AMERICAN MATHEMATICAL SOCIETY \\ CHEVRON RESEARCH CORPORATION \\ NAVAL WEAPONS CENTER
}

The Supporting Institutions listed above contribute to the cost of publication of this Journal, but they are not owners or publishers and have no responsibility for its content or policies.

Mathematical papers intended for publication in the Pacific Journal of Mathematics should be in typed form or offset-reproduced, (not dittoed), double spaced with large margins. Underline Greek letters in red, German in green, and script in blue. The first paragraph or two must be capable of being used separately as a synopsis of the entire paper. The editorial "we" must not be used in the synopsis, and items of the bibliography should not be cited there unless absolutely necessary, in which case they must be identified by author and Journal, rather than by item number. Manuscripts, in duplicate if possible, may be sent to any one of the four editors. Please classify according to the scheme of Math. Rev. Index to Vol. 39. All other communications to the editors should be addressed to the managing editor, Richard Arens, University of California, Los Angeles, California, 90024.

50 reprints are provided free for each article; additional copies may be obtained at cost in multiples of 50 .

The Pacific Journal of Mathematics is published monthly. Effective with Volume 16 the price per volume (3 numbers) is $\$ 8.00$; single issues, $\$ 3.00$. Special price for current issues to individual faculty members of supporting institutions and to individual members of the American Mathematical Society: $\$ 4.00$ per volume; single issues $\$ 1.50$. Back numbers are available.

Subscriptions, orders for back numbers, and changes of address should be sent to Pacific Journal of Mathematics, 103 Highland Boulevard, Berkeley, California, 94708.

PUBLISHED BY PACIFIC JOURNAL OF MATHEMATICS, A NON-PROFIT CORPORATION

Printed at Kokusai Bunken Insatsusha (International Academic Printing Co., Ltd.), 7-17, Fujimi 2-chome, Chiyoda-ku, Tokyo, Japan. 


\section{Pacific Journal of Mathematics}

Vol. 38, No. $2 \quad$ April, 1971

Richard Davis Anderson and Thomas Ashland Chapman, Extending

homeomorphisms to Hilbert cube manifolds .................. 281

Nguyen Huu Anh, Restriction of the principal series of $\operatorname{SL}(n, \mathbf{C})$ to some

reductive subgroups................................ 295

David W. Boyd, Indices for the Orlicz spaces . . . . . . . . . . . . 315

William Garfield Bridges, The polynomial of a non-regular digraph ...... 325

Billie Chandler Carlson, Robert K. Meany and Stuart Alan Nelson, Mixed

arithmetic and geometric means........................ 343

H. A. Çelik, Commutative associative rings and anti-flexible rings ...... 351

Hsin Chu, On the structure of almost periodic transformation groups ...... 359

David Allyn Drake, The translation groups of n-uniform translation

Hjelmslev planes ................................ 365

Michael Benton Freeman, The polynomial hull of a thin two-manifold . . . . 377

Anthony Alfred Gioia and Donald Goldsmith, Convolutions of arithmetic

functions over cohesive basic sequences .................... 391

Leslie C. Glaser, A proof of the most general polyhedral Schoenflies

conjecture possible ................................

Thomas Lee Hayden and Ted Joe Suffridge, Biholomorphic maps in Hilbert

space have a fixed point ................................ 419

Roger Alan Horn, Schlicht mappings and infinitely divisible kernels ...... 423

Norman Ray Howes, On completeness ...................... 431

Hideo Imai, Sario potentials on Riemannian spaces................ 441

A. A. Iskander, Subalgebra systems of powers of partial universal

algebras.

Barry E. Johnson, Norms of derivations of $\mathscr{L}(\mathrm{X})$.

David Clifford Kay and Eugene W. Womble, Axiomatic convexity theory and relationships between the Carathéodory, Helly, and Radon numbers

Constantine G. Lascarides, A study of certain sequence spaces of Maddox

and a generalization of a theorem of Iyer .............

C. N. Linden, On Blaschke products of restricted growth .

John S. Lowndes, Some triple integral equations ................. 515

Declan McCartan, Bicontinuous preordered topological spaces ......... 523

S. Moedomo and J. Jerry Uhl, Jr., Radon-Nikodým theorems for the Bochner and Pettis integrals ...

Calvin Cooper Moore and Joseph Albert Wolf, Totally real representations

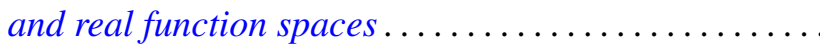

Reese Trego Prosser, A form of the moment problem for Lie groups. ... 